\title{
Intrinsic Autofluorescent Phenotype: A Novel Biomarker to Identify and Isolate Glioma Stem-Like Cells
}

\author{
BIJIA WANG, HAOYI LI ${ }^{1}$, ZHENHUA. ZHOU, KANGNING CHEN AND XUEGANG LI*
}

Department of Neurology, ${ }^{1}$ Department of Neurosurgery, First Affiliated Hospital, Army Medical University, Chongqing 400016, China

Wang et al.: Intrinsic Autofluorescent Phenotype: A Novel Biomarker

\begin{abstract}
Although defined markers such as prominin-1 play a successful role in isolating glioma stem-like cells, their expression is susceptible to environmental alteration. Therefore, we determine the efficiency of the riboflavin-addicted autofluorescence method in isolating glioma stem-like cells. GL261 glioma stem-like cells were selected and the agent of riboflavin was added to the culture medium. Then, glioma stemlike cells with autofluorescence were identified and isolated through flow cytometry. Subsequently, cell counting kit-8 was used to detect the proliferation of targeted cells and we developed a mouse model of xenograft to record the overall survival rate and tumor size and weight per day. Additionally, the expressions of Ki67, proliferating cell nuclear antigen and nestin in the tumor mass were explored by immunohistochemical assay. The feature of autofluorescence in glioma stem-like cells was identified and isolated by flow cytometry. The proliferation and malignancy of the autofluorescence glioma stem-like cells were stronger than glioma stem GL261 cells screened by prominin-1 labeling. Cell counting kit-8 assay demonstrated a significantly stronger proliferation of glioma stem-like cells with autofluorescence $(\mathrm{p}<0.01)$. In vitro, the Kaplan-Meier curve indicated mice bearing fluorescent stem cells had significantly decreased overall survival $(p<0.01)$ and increased tumor volume $(p<0.01)$ and weight $(p<0.01)$. Then, the expression of Ki67, proliferating cell nuclear antigen and nestin in the tumor-derived from fluorescent stem cells was significantly increased by immunohistochemical assay $(\mathbf{p}<\mathbf{0 . 0 5})$. Intrinsic autofluorescent phenotype in glioma stem-like cells were identified and characterized and the marker was used to isolate these cells with high malignancy.
\end{abstract}

Key words: Glioma, riboflavin, autofluorescence, glioma stem-like cells

Glioma originated from epidemic cells is the most common primary malignant brain tumor, which is characterized by invasive growth with dismal outcomes. The conventional therapy includes surgical removal, chemotherapy, radiotherapy etc., however, high recurrence, mortality and rapid progress remain existed $^{[1,2]}$. Currently, there is increasing evidence showing the existence of a tiny minority of tumor cell subtypes equipped with tumorigenic feature in glioma tissue. Due to its shared similarity with stem cells, including the invasive growth in glioma, local recurrence and distant metastasis, the subtype is called glioma stem-like cells $(\mathrm{GSCs})^{[3]}$. They have characteristics of self-renewing, multi-potential differentiation, unlimited proliferation, heterogeneity etc., ${ }^{[1-4]}$. GSCs are tolerated to chemotherapy and radiotherapy, now considered as the important original cause and stimulus for the origin and growth of glioma, and also the source of malignancy. Therefore, various researches on GSCs and development of the targeted drugs are important to the basic research and clinical application. However, the accurate method for identification and screening of GSCs remains currently lacking. The molecular characteristics of tumor stem cells are similar to those of stem cells, so the positive prominin-1 (CD133) marker is considered to be the basis for the screening and identification of various tumor stem cells. Singh et al. ${ }^{[5,6]}$, have separated the $\mathrm{CD}_{133^{+}} \mathrm{GSC}$ from various types of glioma. However, the changes in the microenvironment can regulate the expression level of the marker. Studies have found that CD133, the subtype of glioma cells, has the characteristic of tumor stem cells ${ }^{[7-9]}$. Therefore, the use of CD133 marker in clinical practice has certain limitations in the early detection and prognosis of glioma and the screening of GSCs in basic research. Currently, Miranda-Lorenzo et al. ${ }^{[10]}$ discovered a type of fluorescent glioma stem cells (FGSCs), which cannot

*Address for correspondence

E-mail: 67433433@qq.com 
be affected by the microenvironment and the heterogeneity of the tumor itself. Meanwhile, the experiments in vitro and in vivo have proved that fluorescent cells are significantly higher in malignancy and tumorgenicity than tumor stem cells screened by traditional methods. Glioma stem cells also exist in gliomas. Whether such stem cells are autofluorescent, or whether glioma stem cells can be screened by autofluorescence has not been reported. In this study, autofluorescent cells in the GL261 glioma stem cell line were screened, observed and compared. The results can be used as a unified marker for GSCs and provide a basis for their screening. Furthermore, it can provide new targets for the clinical treatment of gliomas in the future and is significant for the early detection, prevention and prognosis of gliomas. The human malignant glioma GL261 stem cell line $\left(\mathrm{CD} 133^{+}\right)$was provided by the Department of Neurosurgery of our hospital. The cell counting kit-8 (CCK-8) reagent test kit was purchased from Dojindo Chemical Technology Co., Ltd., Japan. The proliferating cell nuclear antigen (PCNA) antibody was purchased from Dako, the Ki67 antibody from Biyuntian Biotechnology Co., Ltd., and the Nestin antibody from Millipore. Severe combined immunodeficiency mouse (SCID mouse), female, 4 to 5 w old, the body mass of 10 to $15 \mathrm{~g}, 40$ in total, were provided by the Animal Test Center of Army Military Medical University and bred according to specific pathogen-free animals standards. GL261 stem cells was prepared and cultivated in the fresh Dulbecco's modified Eagle's medium/Nutrient mixture F-12 medium (containing $2 \% \mathrm{~B} 27,10 \mathrm{ng} / \mathrm{ml}$ recombinant human epidermal growth factor (rhEGF), $10 \mathrm{ng} / \mathrm{ml}$ basic fibroblast growth factor (bFGF), $100 \mathrm{U} / \mathrm{ml}$ penicillin $\mathrm{G}$ and $100 \mu \mathrm{g} / \mathrm{ml}$ streptomycin). The medium was changed every $24 \mathrm{~h}$ until the suspended glioma stem cell pellets stabilized. In order to induce the adherence of stem cells for observation, the suspended stem cell pellets were first dispersed and dissolved in cell digestion solution, then coated with polylysine and cultured in a medium containing $1 \%$ fetal bovine serum (FBS) for phase-contrast microscopic observation. After adding $30 \mu \mathrm{mol} / 1$ riboflavin, the cultured GL261 stem cells was incubated for $12 \mathrm{~h}$ at $37^{\circ}$. A small amount of the suspension was placed on a glass slide and the autofluorescent cell was observed under the fluorescent microscope (BX60, Olympus). The cells were resuspended in the sorting solution $[1 \times$ phosphate buffered saline (PBS); 3 \% FBS (volume fraction); 3 $\mathrm{mmol} / \mathrm{l}$ Ethylenediaminetetraacetic acid (EDTA)], which contained $10^{6}$ cells $/ \mathrm{ml}$. The flow cytometer (BD
FACSCanto ${ }^{\mathrm{TM}}$ II flow cytometer) was used for sorting with the two filters of 530/40 and 580/30 to sort out autofluorescent cells. All animal experiments are implemented according to the laboratory animal standards of the Army Medical University. The cell suspensions of the selected FGSCs and GL261-derived stem cells were prepared for the injection $(10 \mu \mathrm{l} /$ mouse, 104 cells/mouse). All nude mice were divided into the GL261 stem cell group $(n=10)$ and the fluorescent stem cell group $(\mathrm{n}=10)$, in which the former was subcutaneously planted with GL261 stem cells in the groin area and the latter was subcutaneously planted with fluorescent stem cells in the groin area. The long diameter (a) and short diameter (b) of the tumors were measured every $2 \mathrm{~d}$ and the tumor growth volume was calculated with the formula $\mathrm{V}=\mathrm{a} \times \mathrm{b}^{2} / 2\left(\mathrm{~mm}^{3}\right)$. The nude mice were discontinued after $28 \mathrm{~d}$ of rearing and their tumors were taken out and weighed. The rear mice were grouped in the same way as above and the survival conditions of the tumor-bearing mice in two groups were observed. To detect the proliferation ability of FGSCs and GSCs, the CCK-8 kit was used. The suspended neural stem cell pellets were pipetted into single neural stem cells, planted in a 96-well plate (100 $\mu \mathrm{l} / \mathrm{well}, 2 \times 10^{5}$ cells $/ \mathrm{ml}$ ) and incubated for $24 \mathrm{~h}$. Then the medium was added with CCK- 8 solution $(10 \mu \mathrm{l} /$ well) and incubated for $4 \mathrm{~h}$. The Microplate reader (Bio-RAD, Model 680) was used to measure the optical density of each well at a reference wavelength of 450 $\mathrm{nm}$. The removed tumor tissue of the nude mice was embedded in paraffin and sliced with a thickness of 5 $\mu \mathrm{m}$. The slices were sticked on the glass slide for staining. According to the staining principle, PBS containing $0.8 \%$ hydrogen peroxide and containing 0.3 $\%$ Tween-20 and $5 \%$ Bovine serum albumin (BSA) were used to remove endogenous peroxidase activity and block non-specific reactions, respectively. Then, the samples were incubated with the primary antibody (1:200) at $4^{\circ}$ for $12 \mathrm{~h}$, including Ki67 (murine, Beyotime), PCNA (murine, Dako) and Nestin (rabbit source, Millipore). Furthermore, they were incubated with the secondary antibody $(1: 1000)$ at room temperature for $5 \mathrm{~h}$, including anti-mouse-horseradish peroxidase (HRP) (Santa Cruz) and anti-rabbbit-HRP (Santa Cruz); finally the slices were incubated with the addition of avidin-biotin complex (ABC) peroxidase in parallel with diaminobenzidine (DAB) (Boster, China) color reaction to observe the results under a light microscope. The statistical package for the social sciences (SPSS) 16.0 software was used for analysis. The data were expressed as mean \pm standard deviation. 
The student-t test was performed for comparison between two groups. $p<0.05$ is considered statistically significant. To detect the presence of FGSCs in routinely cultured GL261 stem cells, the GL261 stem cell line was cultivated (fig. 1) and then riboflavin was added to the culture medium. FGSCs were found under the microscope (fig. 2). Subsequently, the flow cytometry was used to sort GL261 (CD133 $)$ stem cells and the results showed the FGSCs accounted for $85.21 \%$ of the total while non-fluorescent tumor stem cells accounted for $14.79 \%$. Then, the sorting from the FGSCs showed that $\mathrm{CD} 133^{+}$cells accounted for $72.88 \%$ of the total and CD133- cells accounted for $27.12 \%$. The CCK-8 test found that the proliferation ability of FGSCs was significantly higher than that of GL261 stem cells (fig. $3 \mathrm{~A}, \mathrm{p}<0.01)$. The proliferation ability of FGSCs and GSCs were further compared in the in vivo experiments. The same amount of FGSCs and GSCs were subcutaneously planted in the groin area of the nude mice and the proliferation of subcutaneous tumors in both groups was observed every $2 \mathrm{~d}$. From the $11^{\text {th }} \mathrm{d}$, the tumor volume in the FGSCs group was significantly larger than that in the GSC group (fig. $3 \mathrm{~B}, \mathrm{p}<0.01$ ). After $28 \mathrm{~d}$ of rearing, the mice were discontinued and the tumors were taken out and weighed. The tumor mass of the FGSC group was found significantly larger than that of the GSC group (fig. 3C, $\mathrm{p}<0.01$ ). Subsequently, the survival ability of both groups was compared and the survival ability of the GSC group is significantly higher than that of the FGSC group (fig. $3 \mathrm{D}, \mathrm{p}<0.01)$. To verify the high malignancy of FGSCs, which means the stronger proliferation ability and the stemness of the cells themselves, the antigens of PCNA, Ki67 and Nestin related to cell proliferation and stemness were selected. After removing the tumors of the tumor-bearing mice, paraffin slices were prepared and enzyme-linked immunostaining was processed. It was found that the expressions of PCNA, Ki67 and Nestin in the FGSC group were significantly higher than those in the GSC group (fig. 4A and fig. 4B, $\mathrm{p}<0.05$ ). Effective methods for the treatment of glioma have always been the focus and hotspot. Singh et al. ${ }^{[5,6]}$ isolated glioma stem cells with $\mathrm{CD} 133^{+}$phenotype from different types of glioma, which showed similar markers when compared with normal stem cells. While, GSCs have the feature of heterogeneity and its cell surface markers and functional status are usually affected by the tumor microenvironment ${ }^{[11,12]}$. For example, factors related to vascular endothelial cells can regulate the expression level of CD133 and the glioma stem cell surface marker and affect the glioma stem cells' ability of self-renewing and proliferation ${ }^{[1,13]}$. No correlation was found between the markers and the occurrence or functional characteristics of GSCs. Miranda-Lorenzo et al. ${ }^{[10]}$ have found the presence of the inherent fluorescent phenotypes of certain cell subtypes in some tumors. Such cells have the characteristics of tumor stem cells, of which CD133 cells account for a large proportion while there are still CD133- cells. Several ABCG2-embedded vesicles, containing fluorescent riboflavin, were found in the cytoplasm of these fluorescent cells by molecular and immunology detections. Its expression is an inherent characteristic of fluorescent cells and cannot be affected by the tumor microenvironment and the heterogeneity of the tumor itself. At present, $\mathrm{CD} 133^{+}$glioma cells are believed as the potential stem cells we are targeting but there are also studies showing that CD133- glioma cells still have the potential of being the most malignant. The root cause is the heterogeneity of tumor cells, with no exception in stem cells. The FGSCs in CD133+ phenotype were explored in GL261 glioma, based on the tumor stem cells characteristics of the riboflavinaddicted. This experiment has proved the presence of a certain kind of riboflavin fluorescent stem cells in glioma with a high degree of malignancy without any effect by the external microenvironment and was expressed as an inherent phenotype. It provides new directions and possibilities for the future screening of GSCs and for accurately targeting and killing of GSCs. In this study, GL261 glioma stem cells were first cultured systematically and then the experiment was performed after these cells stabilized into pellets and grown in suspension. The GSCs were cultured with riboflavin and several tumor stem cells with green fluorescence were found under the fluorescence microscope. Screening of $\mathrm{CD} 133^{+}$cells by the flow cytometer found that fluorescent stem cells accounted for higher proportions than non-fluorescent cancer stem cells. This may indicate that there is still a class of tumor cells with low malignancy among the tumor stem cells currently screened. Meanwhile, the screening of fluorescent stem cells found that the proportion of $\mathrm{CD}_{133^{+}}$cells was higher than that of CD133-, indicating that there is still a class of fluorescent stem cells that do not express $\mathrm{CD}_{133^{+}}$. In short, fluorescence may be the inherent phenotype of tumor stem cells and the fluorescence does not change following the change of the tumor microenvironment. This may also indirectly explain and compensate the hypothesis that the CD133may also be the hypothesis of tumor stem cells. Perhaps the introduction of riboflavin can improve the accuracy 
of cancer stem cell screening. To compare the proliferation ability of FGSCs and ordinary GSCs, the CCK-8 kit was used to do the detection within $24 \mathrm{~h}$, which showed that the proliferation ability of FGSCs was significantly greater than that of ordinary GSCs. From this perspective, FGSCs are relatively more malignant. Finally, in vitro experiments and immunohistochemical studies have shown that FGSCs still exist in $\mathrm{CD}_{133^{+}}$tumor stem cells and both the malignant degree and proliferation ability of these stem cells are higher than that of the currently used stem cells screened by the phenotype CD133. This indicates that the $\mathrm{CD} 133^{+}$marker cannot completely screen all the most malignant tumor stem cells, which can be inferred the presence of some high malignant stem tumor cells in CD133- tumor cells. This may be the shortcoming of the current $\mathrm{CD} 133^{+}$targeted therapeutic drugs. In summary, fluorescent tumors stem cells may be the specific phenotype of tumor stem cells, with a relatively high degree of malignancy and do not change with microenvironment changes. Targeting fluorescent tumor stem cells is expected to become an effective screening strategy and at the same time, provides a new target for future clinical targeted therapy. Further study of the mechanism of fluorescence will help us to understand the process of this phenotype more comprehensively and to provide a more sufficient theoretical and experimental basis for clinical treatment.

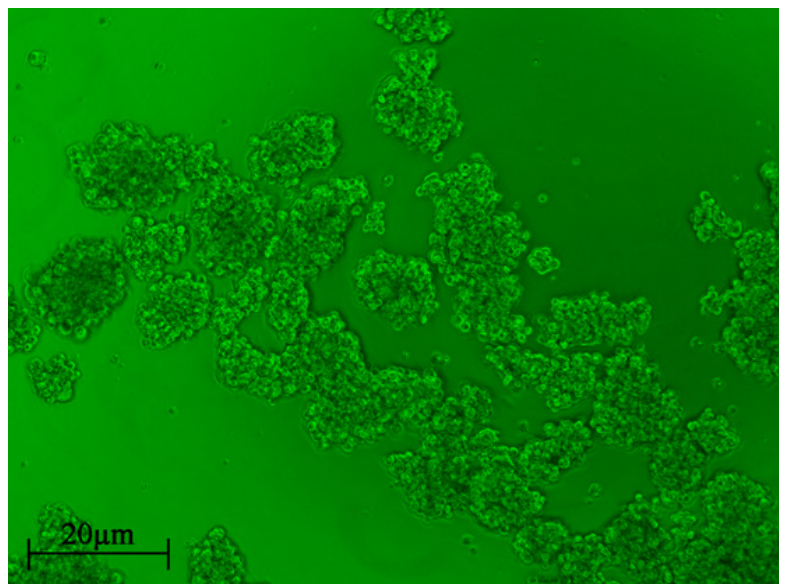

Fig. 1: Morphology of CD133+ GL261 glioma stem cells observed by phase contrast microscope


Fig. 2: Autofluorescence of riboflavin-addicted glioma stem cells under fluorescence microscope 
www.ijpsonline.com

A

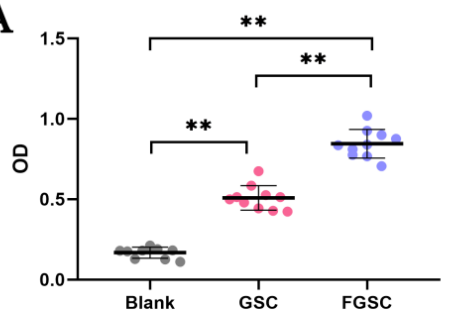

C

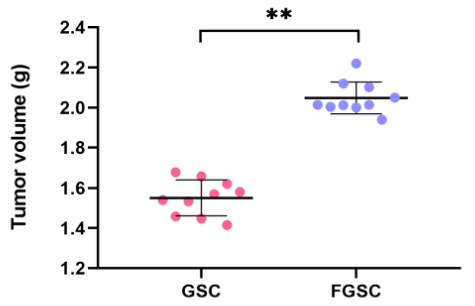

B



D

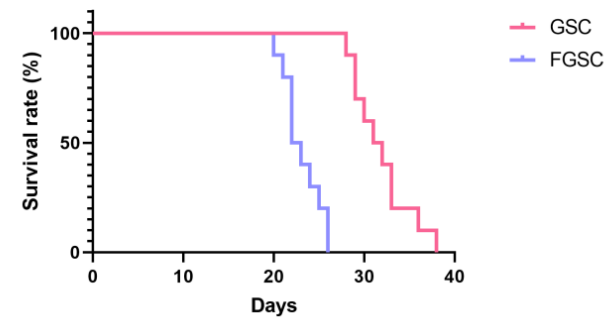

Fig. 3: Proliferation ability of autofluorescence stem cells and survival rate of tumor bearing mice (A) proliferation of fluorescent stem cells and GL261 stem cells examined by CCK-8; (B) Comparison of tumor volume changes between FGSC-derived and GSC-derived tumors; (C) Comparison of tumor weight between FGSC-derived and GSC-derived tumors; (D) Comparison of survival between FGSC-derived and GSC-derived tumors bearing mice. $(-\infty)$ GSC, $(--)$ FGSC, $(-)$ GSC, $(-)$ FGSC

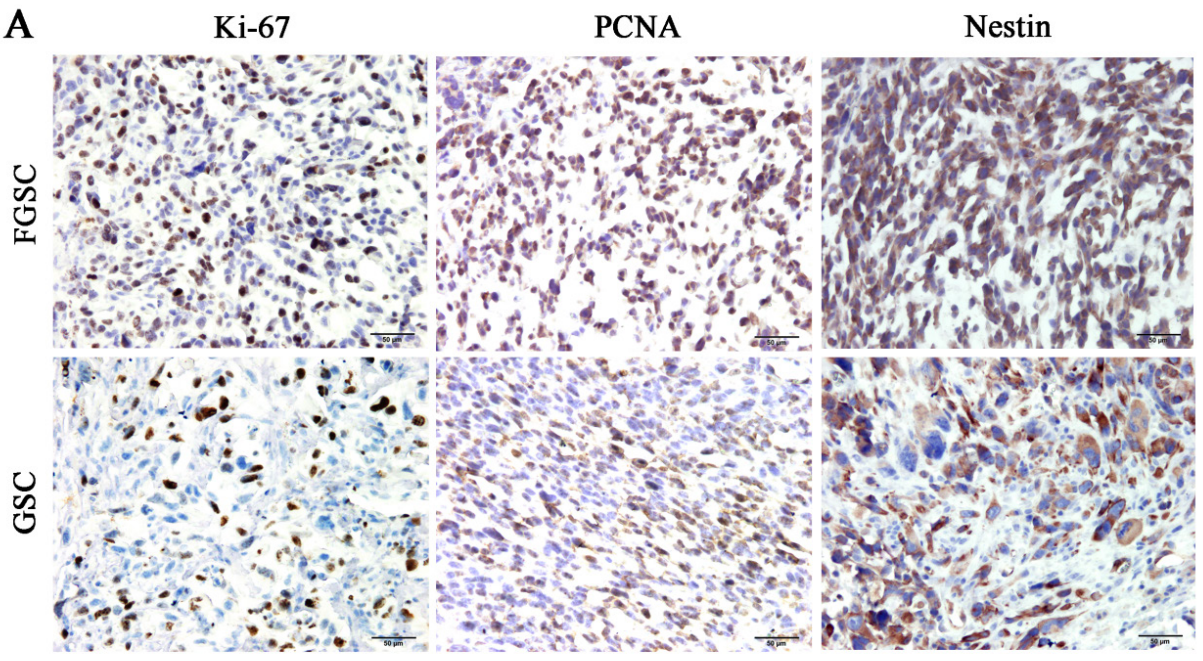

B

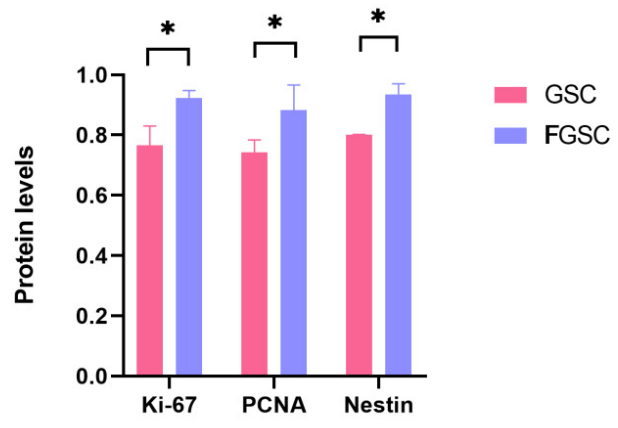

Fig. 4: Expression of PCNA, Ki67 and Nestin in FGSC and GSC examined by immunohistochemistry ( $\mid \square$ ) GSC; ( $\square$ ) FGSC 


\section{Authors' contributions:}

Bijia Wang and Haoyi Li have contributed equally to this work.

\section{Conflicts of interest:}

There are no conflicts of interest.

\section{REFERENCES}

1. Schonberg DL, Lubelski D, Miller TE, Rich JN. Brain tumor stem cells: Molecular characteristics and their impact on therapy. Mol Aspects Med 2014;39:82-101.

2. Ung N, Yang I. Nanotechnology to augment immunotherapy for the treatment of glioblastoma multiforme. J Neurooncol 2015;123(3):473-81.

3. Reya T, Morrison SJ, Clarke MF, Weissman IL. Stem cells, cancer and cancer stem cells. Nature 2001;414(6859):105-11.

4. Donnenberg VS, Donnenberg AD. Multiple drug resistance in cancer revisited: the cancer stem cell hypothesis. J Clin Pharmacol 2005;45(8):872-7.

5. Singh SK, Clarke ID, Terasaki M, Bonn VE, Hawkins C, Squire $\mathrm{J}$, et al. Identification of a cancer stem cell in human brain tumors. Cancer Res 2003;63(18):5821-8.

6. Singh SK, Hawkins C, Clarke ID, Squire JA, Bayani J, Hide $\mathrm{T}$, et al. Identification of human brain tumour initiating cells. Nature 2004;432(7015):396-401.
7. Clément V, Dutoit V, Marino D, Dietrich PY, Radovanovic I. Limits of CD133 as a marker of glioma self-renewing cells. Int J Cancer 2009; 125(1):244-8.

8. Hill RP. Identifying cancer stem cells in solid tumors: case not proven. Cancer Res 2006;66(4):1891-6.

9. Kern SE, Shibata D. The fuzzy math of solid tumor stem cells: a perspective. Cancer Res 2007;67(19):8985-8.

10. Miranda-Lorenzo I, Dorado J, Lonardo E, Alcala S, Serrano AG, Clausell-Tormos J, et al. Intracellular autofluorescence: a biomarker for epithelial cancer stem cells. Nat Methods 2014;11(11):1161-9.

11. Lathia JD, Gallagher J, Heddleston JM, Wang J, Eyler CE, MacSwords J, et al. Integrin alpha 6 regulates glioblastoma stem cells. Cell Stem Cell 2010;6(5):421-32.

12. Soeda A, Park M, Lee D, Mintz A, Androutsellis-Theotokis $\mathrm{A}$, McKay RD, et al. Hypoxia promotes expansion of the CD133 positive glioma stem cells through activation of HIF$1 \alpha$. Oncogene 2009;28(45):3949-59.

13. Calabrese C, Poppleton H, Kocak M, Hogg TL, Fuller C, Hamner B, et al. A perivascular niche for brain tumor stem cells. Cancer Cell 2007;11(1):69-82.

This is an open access article distributed under the terms of the Creative Commons Attribution-NonCommercial-ShareAlike 3.0 License, which allows others to remix, tweak, and build upon the work non-commercially, as long as the author is credited and the new creations are licensed under the identical terms

This article was originally published in a special issue, "Diagnostic and Therapeutic Advances in Biomedical Research and Pharmaceutical Sciences"

Indian J Pharm Sci 2021:83(5) spl issue "33-38" 